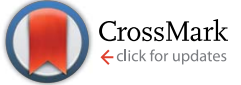

Cite this: J. Mater. Chem. A, 2016, 4, 12790

Received 5th June 2016

Accepted 12 th July 2016

DOI: 10.1039/c6ta04700a

www.rsc.org/MaterialsA

\title{
PDMS membranes containing ZIF-coated mesoporous silica spheres for efficient ethanol recovery via pervaporation $\uparrow$
}

\author{
Parimal V. Naik, ${ }^{a}$ Lik H. Wee, ${ }^{\text {*a }}$ Maria Meledina, ${ }^{\text {b }}$ Stuart Turner, ${ }^{\text {b }}$ Yanbo Li, ${ }^{a}$ Gustaaf Van \\ Tendeloo, $^{\text {b Johan A. Martens }}{ }^{a}$ and Ivo F. J. Vankelecom*a
}

\begin{abstract}
The design of functional micro- and mesostructured composite materials is significantly important for separation processes. Mesoporous silica is an attractive material for fast diffusion, while microporous zeolitic imidazolate frameworks (ZIFs) are beneficial for selective adsorption and diffusion. In this work, ZIF-71 and ZIF-8 nanocrystals were grown on the surface of mesoporous silica spheres (MSS) via the seeding and regrowth approach in order to obtain monodispersed MSS-ZIF-71 and MSS-ZIF- 8 spheres with a particle size of $2-3 \mu \mathrm{m}$. These MSS-ZIF spheres were uniformly dispersed into a polydimethylsiloxane (PDMS) matrix to prepare mixed matrix membranes (MMMs). These MMMs were evaluated for the separation of ethanol from water via pervaporation. The pervaporation results reveal that the MSS-ZIF filled MMMs substantially improve the ethanol recovery in both aspects viz. flux and separation factor. These MMMs outperforms the unfilled PDMS membranes and the conventional carbon and zeolite filled MMMs. As expected, the mesoporous silica core allows very fast flow of the permeating compound, while the hydrophobic ZIF coating enhances the ethanol selectivity through its specific pore structure, hydrophobicity and surface chemistry. It can be seen that ZIF- 8 mainly has a positive impact on the selectivity, while ZIF-71 enhances fluxes more significantly.
\end{abstract}

\section{Introduction}

Mixed matrix membranes (MMMs), prepared by combining the favourable features of polymers and inorganic nanoparticles, have proven to be promising materials for membrane based separations. These MMMs are composed of porous particles; such as zeolites, ${ }^{\mathbf{1 - 7}}$ silicas, ${ }^{\mathbf{8}, \mathbf{9}}$ or carbonaceous particles, ${ }^{\mathbf{1 0 , 1 1}}$ dispersed in a polymer matrix. However, membranes filled with these particles possess some issues which limit their practical application. The main issue is defect-formation in the membranes during the membrane preparation, due to (i) fillerpolymer incompatibility and (ii) agglomeration of the fillers, resulting in an inhomogeneous dispersion within the polymer matrix, especially at higher filler loadings. ${ }^{\mathbf{1 2}-15}$ Therefore, further development of new, efficient porous fillers is required in order to prepare high-flux, selective and defect-free MMMs.

Metal-organic frameworks (MOFs), a new class of crystalline porous materials constructed from inorganic metal ions and

${ }^{a}$ Centre for Surface Chemistry and Catalysis, KU Leuven, Celestijnenlaan 200f, B3001, Post box 2461, Heverlee, Leuven, Belgium.E-mail: likhong.wee@biw.kuleuven.be; ivo. vankelecom@biw.kuleuven.be

${ }^{b}$ Electron Microscopy for Materials Science, University of Antwerp, Groenenborgerlaan 171, B2020, Antwerp, Belgium

† Electronic supplementary information (ESI) available. See DOI: 10.1039/c6ta04700a organic linkers have emerged as potential fillers for the fabrication of MMMs. ${ }^{16-25}$ MOFs offer great advantages over other conventional inorganic porous fillers, e.g. zeolites or carbonaceous particles, mostly due to: (i) better compactibility of the polymer chains with MOFs due to their partial organic nature, and (ii) tunable hydrophobicity by selection of appropriate ligands in the synthesis of MOFs. Within the MOF family, zeolitic imidazolate frameworks (ZIFs) are porous crystals with extended 3D structures constructed from tetrahedral metal ions (e.g. $\mathrm{Zn}$ ) bridged by imidazolate linkers (Im). ${ }^{26}$ ZIFs exhibit permanent porosity and high thermal and chemical stability, which make them attractive candidates for many applications such as separation and gas storage. ${ }^{26-28}$ Over the last decade, more than 90 different ZIF-topologies have been reported. ${ }^{26}$ Among them, ZIF-8 is one of the most extensively studied ZIF species. It has a sodalite (SOD) topology with a small pore window of $3.4 \AA^{27}$ The outstanding thermal stability and hydrophobic nature of the ZIF-8 are ideally suited for preparing MMMs which are applicable in solvent resistant nanofiltration (SRNF), ${ }^{28,29}$ gas separation, ${ }^{30-37}$ and pervaporation. ${ }^{38-41}$ For instance, simultaneous spray self-assembly of a ZIF-8-PDMS nanohybrid membrane with high loading (40 wt\%) showed excellent biobutanol-selective pervaporation performance. $^{38}$ ZIF-8/PBI MMMs have been prepared for pervaporation to dehydrate ethanol, isopropanol (IPA) and butanol. ${ }^{39}$ ZIF-71 is another hydrophobic material which possesses a RHO topology with a pore window size of $4.8 \AA^{4.4-45}$ It has been reported 
that ZIF-71 is a potential filler to separate bioalcohols from water through pervaporation. ${ }^{\mathbf{4 6}-49}$ For instance, ZIF-71 filled polyetherblock-amide (PEBA) MMMs were reported for biobutanol recovery from an acetone-butanol-ethanol (ABE) fermentation broth by pervaporation. In addition to an excellent separation, the membrane exhibited a stable performance in the real $\mathrm{ABE}$ fermentation broth over the course of more than 100 hours. $^{47}$

In recent years, micro- and mesostructured composite nanomaterials have attracted a great deal of scientific attention as promising candidates for e.g. catalysis, ${ }^{\mathbf{5 0}}$ photoluminescence,${ }^{51}$ drug delivery, ${ }^{52}$ separations,${ }^{53}$ electrode materials $^{54}$ and fuel cells ${ }^{55}$ owing to their attractive physical characteristics, such as low density, high specific surface area, delivering ability and high surface permeability. The objective of micro- and mesostructured design is to combine the benefits of a microporous shell, e.g. ZIFs possessing a high adsorption capacity, and a mesoporous core, e.g. mesoporous silica, which is expected to facilitate faster diffusion resulting in enhanced separation capacity. Therefore, the combination of micro- and mesostructured composite material is expected to improve the overall separation process with respect to an improved flux and separation factor. Seed coating and chemical modification are effective methods for MOFs coatings. ${ }^{56-60}$ For example, Coronas and co-workers have reported the preparation of MSS-ZIF-8 core-shell spheres via in situ seeding and secondary growth for the preparation of MMMs for the dehydration of ethanol. ${ }^{59,61}$

In present study, uniform and monodispersed MSS-ZIF-71 and MSS-ZIF-8 spheres were synthesised. The synthesis method used to prepare MSS-ZIF-71 has been modified via controlling the crystallization kinetic and solvent mediated secondary growth synthetic approach. These MSS-ZIF-71 and MSS-ZIF-8 spheres were used for the preparation of PDMS-based MMMs and evaluated for the separation of ethanol from water via pervaporation. The pervaporation studies using these coreshell fillers provide insight into the effect of two different ZIF framework topologies as a shell forming material on the bioethanol recovery performance.

\section{Experimental}

\section{Chemicals}

Sodium metasilicate $\left(\mathrm{Na}_{2} \mathrm{SiO}_{3}\right)$, cetyltrimethyl ammonium bromide (CTABr) and 2-methylimidazole were purchased from Acros, while zinc nitrate hexahydrate, hexane, methanol, dimethyl formamide (DMF) and zinc acetate were purchased from Sigma-Aldrich. 4,5-Dichloroimidazole was purchased from Alfa Aesar, ethyl acetate from VWR, polydimethylsiloxane (PDMS) RTV-615 from GE silicones (Belgium).

\section{Synthesis of mesoporous silica sphere (MSS)}

MSS were prepared according to the synthesis procedure described by Sorribas et al. ${ }^{59}$ The synthesis mixture has a molar concentration of $1.5 \mathrm{Na}_{2} \mathrm{SiO}_{3}: 1 \mathrm{CTABr}: 361 \mathrm{H}_{2} \mathrm{O}: 7.4 \mathrm{CH}_{3}$ $\mathrm{COOC}_{2} \mathrm{H}_{5}$. CTABr and $\mathrm{Na}_{2} \mathrm{SiO}_{3}$ were dissolved in distilled water. Ethyl acetate was then added under stirring for $30 \mathrm{~s}$. After mixing the reactants, the solution was kept undisturbed for $5 \mathrm{~h}$, followed by heating at $90{ }^{\circ} \mathrm{C}$ for $50 \mathrm{~h}$. The resulting white precipitate was filtered, washed with water and ethanol, dried overnight at $60{ }^{\circ} \mathrm{C}$ and calcined in a muffle oven under a $\mathrm{N}_{2} / \mathrm{O}_{2}$ flow at $600{ }^{\circ} \mathrm{C}$ for $8 \mathrm{~h}$ with a heating ramp of $0.5{ }^{\circ} \mathrm{C} \mathrm{min}^{-1}$.

\section{Preparation of ZIF coated mesoporous silica spheres (MSS- ZIF)}

The synthesis of MSS-ZIF-8 spheres was carried out as reported by Coronas and co-workers. ${ }^{59}$ The method consists of in situ seeding and secondary crystal growth. The seeding process is required to obtain MSS with a homogeneous and completely covered ZIF layer. The details of the linker and the solvents used for the ZIF nanocrystal formation are listed in Table 1.

Coating of ZIF-71 nanoparticles on MSS spheres was carried out according to the recipe reported by Wee et al. ${ }^{49}$ DMF was used for both seeding and secondary growth synthesis. Reaction condition for MSS-ZIF-71 were optimized by varying different solvent ratios and reaction time.

Seeding process. A molar ratio of $\mathrm{Zn}:$ imidazole $=1: 70$ was used. $0.1 \mathrm{~g}$ of MSS was mixed with $3.78 \mathrm{~g}$ of the respective imidazole linker in $13.3 \mathrm{~g}$ of solvent. Additionally, another $0.1 \mathrm{~g}$ of MSS was mixed with $0.195 \mathrm{~g}$ of the respective zinc salt in $1.3 \mathrm{~g}$ of solvent. Both solutions were stirred and sonicated for $10 \mathrm{~min}$. The zinc salt solution was then mixed with the imidazole solution under stirring for $10 \mathrm{~min}$. The product was collected by centrifugation, washed and dried overnight.

Secondary growth of MSS-ZIFs spheres. $0.14 \mathrm{~g}$ of the seeded MSSs and $1 \mathrm{~g}$ of the imidazole linker were dissolved in $20 \mathrm{~mL}$ of the respective solvent. Additionally, 0.47 of zinc salt was dissolved in $10 \mathrm{~mL}$ of the respective solvent (molar ratio of $\mathrm{Zn}$ : imidazole $=1: 7.6$ ). These two solutions were stirred and sonicated separately for $10 \mathrm{~min}$ followed by vigorous stirring (Table 1). The final product was recovered by centrifugation, washed many times with solvent and dried overnight.

\section{Preparation of mixed matrix membranes (MMMs)}

To prepare the MMMs, two components of the PDMS (RTV-615 A and B, prepolymer and cross-linker, respectively) were dissolved separately in hexane [Fig. S1†]. The MSS-ZIF nanoparticles were dispersed ultrasonically in hexane for $1 \mathrm{~h}$. The

Table 1 ZIF nanoparticle synthesis compositions

\begin{tabular}{llll}
\hline ZIF & Zn source & Imidazole linker & Solvents \\
\hline ZIF-8 & $\mathrm{Zn}\left(\mathrm{NO}_{3}\right)_{2}$ & 2-Methyl imidazole & $\begin{array}{l}\mathrm{H}_{2} \mathrm{O} \text {-seeding } \mathrm{H}_{2} \mathrm{O} / \text { methanol- } \\
\text { growth }\end{array}$ \\
ZIF-71 & $\mathrm{Zn}\left(\mathrm{CH}_{3} \mathrm{COO}\right)_{2}$ & 4,5-Dichloroimidazole & DMF for seeding and growth
\end{tabular}


prepolymer, the cross-linker and the filler were then mixed and stirred at $60{ }^{\circ} \mathrm{C}$ for $4 \mathrm{~h}$ in order to partially crosslink the solution and to obtain a reasonable viscosity. The filler fraction is expressed in weight percent:

Filler fraction $=$

$$
\frac{(\text { weight of sphere })}{(\text { weight of sphere })+(\text { weight of polymer })} \times 100(\text { wt } \%)
$$

The resulting pre-polymerized PDMS solution was poured into a glass petri dish and covered with a funnel to allow controlled evaporation of the solvent. The flat bottomed petri dish was kept in an oven at $110{ }^{\circ} \mathrm{C}$ for at least $1 \mathrm{~h}$ in order to complete the crosslinking process. After crosslinking, the membrane was detached from the petri dish and stored in a dust-free environment. Membranes with different PDMS : filler ratios were prepared. Unfilled PDMS membranes were prepared for comparison.

\section{Characterization of MSS-ZIF spheres and MMMs}

Scanning electron microscopy (SEM) micrographs were obtained from a Philips XL 30 FEG-SEM acquired at $10.0 \mathrm{kV}$. Powder samples were dispersed initially on the carbon tape followed by coating a 1.5-2 $\mathrm{nm}$ thick gold layer to reduce sample charging under the electron beam. SEM micrographs of the membrane cross-sections were obtained by breaking membranes while submerged in liquid nitrogen. $\mathrm{N}_{2}$ physisorption isotherms were obtained using a Micromeritics TriStar II (Micromeritics instrument Corporation, Norcross, Georgia). The measurements were performed at $-196{ }^{\circ} \mathrm{C}$ and all samples were degassed at $200{ }^{\circ} \mathrm{C}$ for $10 \mathrm{~h}$ under nitrogen flow prior to analysis. The pore diameters $(\mathrm{nm})$ were calculated from the adsorption branches of the nitrogen isotherms using the Barrett-Joyner-Halenda (BJH) model. Powder XRD was performed with a Philips high throughput STOE stadi P diffractometer (flat plate sample holder, Bragg-Bretano geometry) using $\mathrm{Cu} \mathrm{K} \alpha_{1}$ radiation $(\lambda=1.5418 \AA$ ). HAADF-STEM imaging and EDX mapping was carried out on a FEI Tecnai Osiris microscope, operated at $200 \mathrm{kV}$ and equipped with a wide solid angle "super-X" EDX detector. The convergence semi-angle used was $10 \mathrm{mrad}$, the inner ADF detection angle was $68 \mathrm{mrad}$.

\section{Pervaporation}

The cross-flow pervaporation set-up used in the experiment is presented in [Fig. $\mathrm{S} 2 \dagger$ ]. The set-up consists of 3 cells in series to hold the membranes. A feed pump is used to circulate the feed through the cells at a speed of $1 \mathrm{~L} \mathrm{~min}^{-1}$ in order to avoid concentration polarization. The temperature was kept constant at $40{ }^{\circ} \mathrm{C}$. During pervaporation, a constant vacuum $(<1 \mathrm{mbar})$ was applied on the permeate side to ensure a constant driving force for the separation. The active membrane area was $0.001589 \mathrm{~m}^{2}$. Membranes were allowed to equilibrate for at least $12 \mathrm{~h}$ before sample collection was initiated. The samples were collected per $3 \mathrm{~h}$. Experiments were carried out with 6 wt $\%$ aqueous ethanol. This concentration was found to be a good representation of the ethanol concentration in the feed used by other researchers in similar separations of alcohol : water mixtures to allow good comparison. ${ }^{62-65}$ Obtained permeates were collected as a function of time in round bottom glass containers using liquid nitrogen in a dewar flask as cooling trap. The concentration of ethanol in the permeate samples was determined using a refractometer (ATAGO RX-7000 $\alpha$ ).

Total flux $(J)$ was calculated by using eqn (1):

$$
J=\frac{m}{A t}
$$

where, $m=$ mass (kg); $A=$ active membrane area $\left(\mathrm{m}^{2}\right) ; t=$ time (h). Partial fluxes were calculated based on the component concentrations in the permeate. Fluxes were normalized to a thickness of $10 \mu \mathrm{m}$.

Separation factor of the membrane was calculated by using eqn (2):

$$
\beta=\frac{\left(\frac{X_{\mathrm{A}}}{X_{\mathrm{B}}}\right)_{\text {permeate }}}{\left(\frac{X_{\mathrm{A}}}{X_{\mathrm{B}}}\right)_{\text {feed }}}
$$

where $X=$ weight fraction; $\mathrm{A}=$ ethanol and $\mathrm{B}=$ water. Three replicates of each $\mathrm{MMM}$ were prepared and measured in parallel. The permeate samples were collected twice after overnight equilibration for each membrane and an average value with standard deviation was calculated out of these 6 observations.

\section{Sorption measurements}

Liquid sorption in the membranes was determined on membrane strips at room temperature. The membrane strips were dried in the oven at $120{ }^{\circ} \mathrm{C}$ before measuring the sorption by immersion in the pure liquids for at least $24 \mathrm{~h}$. The amount adsorbed was determined by weight. The membrane surface was wiped quickly before weighing so as to minimize evaporation of the liquids.

\section{Results and discussion}

\section{MSS-ZIF preparation and characterization}

Fig. 1a shows a SEM image of the calcined MSS with an average particle size of $2.2 \mu \mathrm{m}$. Rough external surface of the MSS makes them an ideal core template for the coating of the ZIF nanocrystals. Fig. $1 \mathrm{~b}$ and $\mathrm{c}$ shows that MSS are covered fully by ZIF-8 nanocrystals with a particle size of $\sim 2.4 \mu \mathrm{m}$ which confirms the successful growth of ZIF-8 on the surface of the MSS.

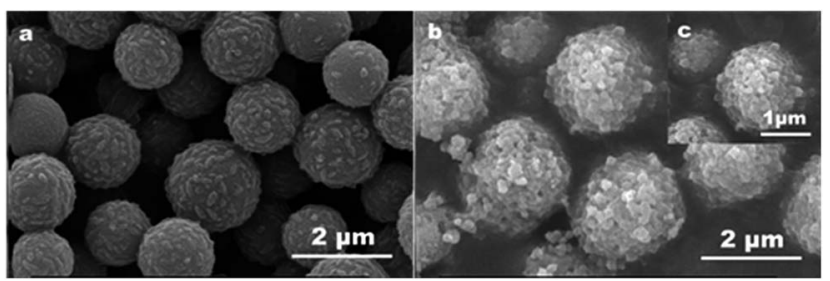

Fig. 1 SEM micrographs of (a) calcined MSS, and (b) \& (c) calcined MSS-ZIF-8. 
ZIF-71 is a three-dimensional porous material with accessible cages of $1.68 \mathrm{~nm}$, interconnected through small windows of $0.48 \mathrm{~nm}$. Recent molecular simulation and experimental results have confirmed the potential of ZIF-71 in selective adsorption of ethanol from aqueous solution. ${ }^{45}$ To improve the pervaporation performance of the mixed matrix membranes, MSS-ZIF-71 core-shell spheres were thus synthesized by a similar method used for the preparation of MSS-ZIF-8. Unfortunately, preparation of monodispersed and homogeneous MSS-ZIF-71 core-shell spheres was not successful due to the simultaneous bulk crystallization and aggregation of the ZIF-71 nanoparticles in methanol, which can be observed in the SEM image shown in Fig. 2a. The particle size of ZIF-71 is relatively large in the range of $600-900 \mathrm{~nm}$. This could be attributed to the different crystallization kinetics of ZIF-8 and ZIF-71. ${ }^{49,66-68}$ ZIF-71 can be synthesized either in methanol or DMF. ${ }^{4,46}$ Recently, a mixed-solvent approach was reported, elucidating the influence of the solvent on the crystallization kinetics and fine tuning of the ZIF-71 particle size. ${ }^{49}$ Further experiments were thus performed by replacing $1 / 3$ volume of methanol by DMF in order to obtain homogeneous growth of ZIF-71 nanoparticles on the surface of the MSS. From Fig. 2b it can be seen that some side-crystallization is still present, but the particle size of the bulk ZIF-71 crystals was significantly reduced. At a higher volume ratio of DMF to methanol (2/3), side aggregation of ZIF-71 was reduced while a more homogeneous growth of ZIF-71 on the MSS was observed, as evidenced from the SEM images in Fig. 2c. With only DMF as solvent, smooth and monodispersed MMS-ZIF-71 spheres were obtained without any ZIF-71 side crystallization (Fig. 2d and e). The ZIF71 nanocrystals forming the shell layer were much smaller than those crystallized in the bulk, mainly due to the steric hindrance induced by the competitive growth of the ZIF-71 crystals in the shell with respect to the bulk crystallization. ${ }^{69}$ By increasing the synthesis time to $4 \mathrm{~h}$, homogeneous MSS-ZIF-71 core-shell spheres were obtained having a particle size of approximately $2.3 \mu \mathrm{m}$ (Fig. 2e). ZIF-71 nanocrystals without a defined morphology were observed. ${ }^{70}$ A prolonged synthesis time $(24 \mathrm{~h})$ resulted in a cracked ZIF-71 shell layer, as shown in Fig. 2f. The

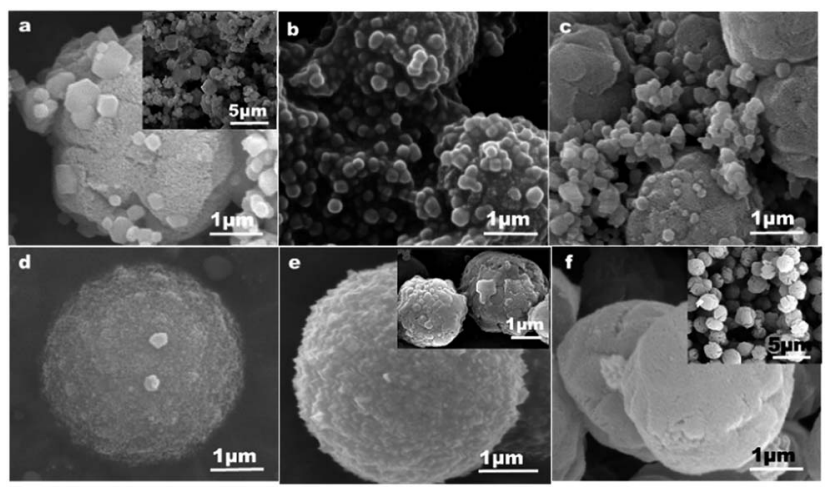

Fig. 2 SEM micrographs of MSS-ZIF-71 viewed at different magnitude as synthesized in different solvent media. (a) Methanol, $2 \mathrm{~h}$, (b) methanol-DMF (2 : 1), $2 \mathrm{~h}$, (c) methanol-DMF (1: 2), $2 \mathrm{~h}$, (d) DMF 2 h, (e) DMF, $4 \mathrm{~h}$, (f) DMF, $24 \mathrm{~h}$. results suggest that DMF effectively reduces the surface free energy on the outer surface of the MSS core allowing spontaneous self-nucleation followed by controlled growth of ZIF-71 nanocrystals. ${ }^{71,72}$ Interactions of solvents with the silica material are expected to be more pronounced when the dipole moment of the solvent molecules is larger. DMF with an exceptionally high dipole moment of $3.82 \mathrm{D}$, is expected to promote the interaction with the surface of the MSS. ${ }^{73}$ Moreover, the highly polar DMF, with a high dielectric constant of 38, could dissolve charged species, in this case the imidazolate nucleophile in the solution, making them freely available in the DMF reservoir for reactive interaction with the MSS surfaces prior to zinc metal coordination. As the availability of primary building units for ZIF motif formation is greatly dependent on their solvation, it is very likely that the solvent composition influences the nucleation kinetics of ZIF-71. In our system, the nucleation of ZIF-71 is enhanced due to stronger solvation of the $\mathrm{Zn}$ (II) cations by DMF with increasing DMF content in a methanol-DMF mixture. ${ }^{49,74}$ Similar crystallization behaviour kinetics have been documented for ZIF-8 synthesis. ${ }^{74}$

In order to further confirm the coating of ZIF-71 on the surface of MSS spheres, the composite material was characterized by HAADF-STEM and EDX analysis. The HAADF-STEM overview image (Fig. 3a) and the corresponding EDX elemental maps for $\mathrm{Si}, \mathrm{O}, \mathrm{Zn}$ and $\mathrm{CI}$ are shown in Fig. 3b-d. As can be seen in Fig. $3 \mathrm{~b}$ and $\mathrm{d}$, both zinc (green) and chloride (blue) are uniformly distributed on the surface of MSS spheres. Both Zn and $\mathrm{Cl}$ originate from the $\mathrm{Zn}$ coordinated 4,5-dichloroimidazolate linkers, indicating the presence of ZIF-71 nanoparticles on the surface of MSS.

BJH pore size distribution shows that after ZIF coating, small mesopores of the MSS get sealed while large mesopores get hindered. It thus confirms the formation of a microporous shell

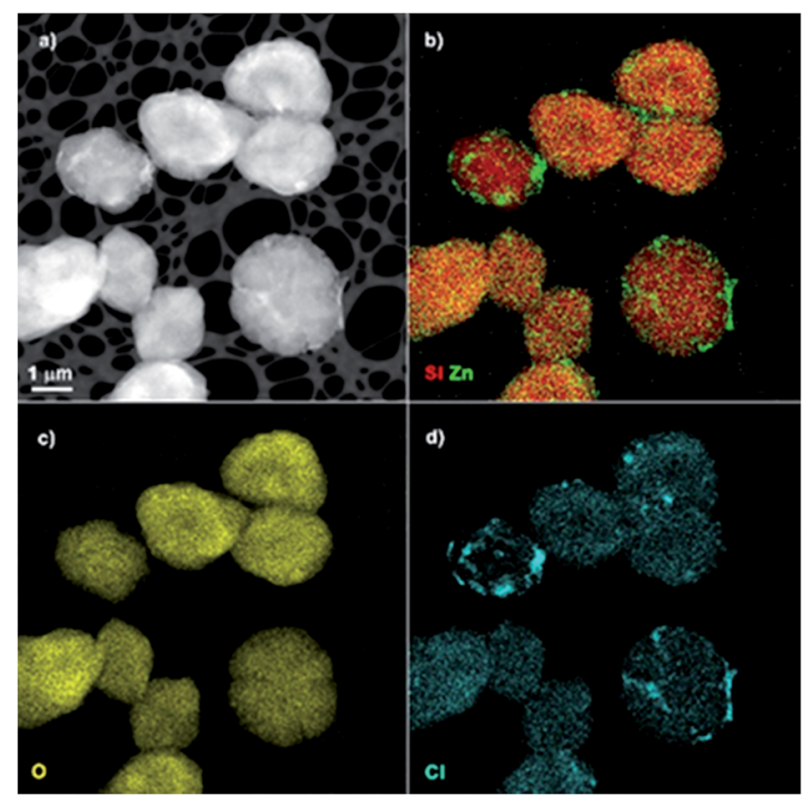

Fig. 3 (a) HAADF-STEM overview image showing a MSS-ZIF-71 spheres together with EDX elemental maps for (b) Si and Zn, (c) O and (d) $\mathrm{Cl}$. 


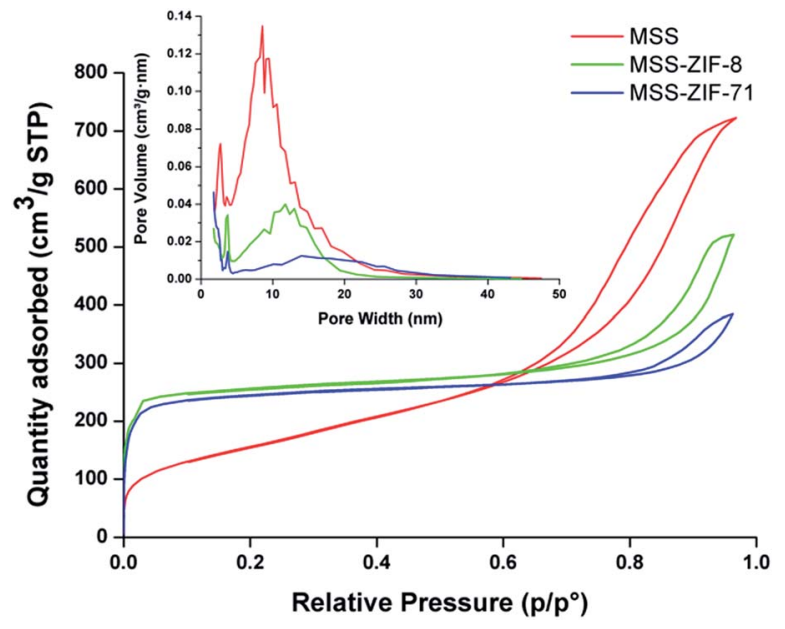

Fig. $4 \quad \mathrm{~N}_{2}$ adsorption-desorption isotherms and the inset shows the $\mathrm{BJH}$ pore size distribution of MSS, MSS-ZIF-8 and MSS-ZIF-71 materials.

of ZIF which might have blocked the accessibility of the nitrogen towards the internal pores of the MSS core. ${ }^{59,75} \mathrm{~A}$ similar phenomenon has also been reported in the case of MSSZIF-8 (ref. 59) and MSS-zeolite. ${ }^{75}$ Nevertheless, a considerable pore volume of the MSS-ZIFs materials is preserved. The total pore volumes of MSS-ZIF-8 and MSS-ZIF-71 materials were 0.75 and $0.59 \mathrm{~cm}^{3} \mathrm{~g}^{-1}$, respectively versus $1.12 \mathrm{~cm}^{3} \mathrm{~g}^{-1}$ for the MSS material. Based on $t$-plot method, the calculated micropore volumes for ZIF-8 and ZIF-71 were 0.34 and $0.30 \mathrm{~cm}^{3} \mathrm{~g}^{-1}$, respectively. The presence of both Type I and IV isotherms for MSS-ZIF-8 and MSS-ZIF-71 materials further confirms the preserved mesostructured silica core and newly formed microstructured ZIF coatings, respectively. ${ }^{76-79}$

The XRD patterns of MSS, MSS-ZIF-71, ZIF-71, MSS-ZIF-8 and ZIF-8 are presented in Fig. 5a. The characteristic peaks of ZIF-71 and ZIF-8 are observed in the XRD patterns of MSS-ZIF71 and MSS-ZIF-8 respectively, which further confirm the presence of an extra-crystalline phase due to the deposition of ZIF nanocrystals on the MSS. ${ }^{48,59}$ The FT-IR spectra of MSS, MSS-ZIF-8, MSS-ZIF-71, ZIF-71 and ZIF-8 are presented in Fig. 5b. For MSS, the peak at $1069 \mathrm{~cm}^{-1}$ can be assigned to the $\mathrm{Si}-\mathrm{O}-\mathrm{Si}$ bonds and a broad peak at $3400-3600 \mathrm{~cm}^{-1}$ is due to unreacted surface silanol groups on the outer surface of the MSS. This peak shows a considerable reduction in the case of

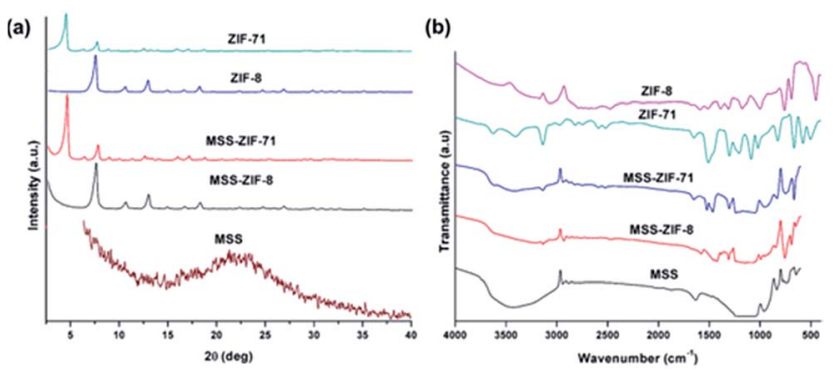

Fig. 5 (a) XRD diffraction patterns and (b) FT-IR spectra of MSS-ZIF spheres and their respective constituting compounds as reference.
MSS-ZIF-71 and MSS-ZIF-8. For MSS-ZIF-71, the characteristic peaks appear at $665 \mathrm{~cm}^{-1}$ (C-Cl vibration) and $1050 \mathrm{~cm}^{-1}(\mathrm{C}-\mathrm{N}$ vibration), while for MSS-ZIF-8, a peak appears at $1145 \mathrm{~cm}^{-1}$ (C-N vibration). These results confirm that the MSS are coated with ZIF-8 and ZIF-71 crystals. $^{48}$

\section{Membrane characterization}

The MMMs were prepared using the MSS-ZIF-71 spheres with a size of $2.3 \mu \mathrm{m}$. In another series of experiments, MSS-ZIF-8 filled MMMs were prepared to enable direct comparison of the pervaporation performance of both fillers. Fig. 6 shows the SEM images of the MSS-ZIF-71 filled MMMs with different loadings. The SEM images reveal that the MSS-ZIF spheres were homogeneously dispersed in the PDMS matrix. No particle agglomeration was observed, even at higher magnifications. The results indicate a good compatibility between the filler and the polymer. The MSS-ZIF-8 filled MMMs are of similar quality (Fig. S3†). Some small voids can be observed between the filler and polymer at higher magnifications. This is possibly due to the removal of residual solvent during membrane preparation at a moment when the PDMS has already crosslinked to a large extent, or while fracturing of the membrane samples in liquid nitrogen when preparing SEM samples.

\section{Membrane swelling}

Fig. 7 shows the sorption tendency for unfilled, as well as for MSS or MSS-ZIF-filled PDMS membranes. In the case of water, all membranes restrict sorption, confirming the hydrophobic nature of the materials. PDMS on its own has a pronounced affinity for ethanol, as reflected by its swelling. When the fillers are incorporated, all sorption values decrease by at least 2 orders of magnitude, clearly indicating a very strong fillerPDMS interaction, most probably even with a certain intrusion of polymer inside the filler. Among the fillers, incorporation of uncoated MSS still allows the largest liquid sorption, in line with its larger free pore volume (Fig. 4). Nevertheless, it should be mentioned that the sorption values are all very low for the filled membranes, and thus the experimental error is relatively high. The calculated standard deviation was $\pm 30 \%$.

\section{Pervaporation performance}

The performance of MSS-ZIF, reference MSS and ZIF filled PDMS membranes with different loadings (10, 15 and $20 \mathrm{wt} \%$ ) was studied (Fig. 8) for the separation of a $6 \%$ aqueous ethanol at $40{ }^{\circ} \mathrm{C}$. All fluxes were normalized to a membrane thickness of $10 \mu \mathrm{m}$.

The incorporation of the MSS into the PDMS network significantly improved the membrane flux as compared to the unfilled PDMS membranes. However, no improvement in the separation factor was observed. The incorporation of MSS enhances the sorption capacity of the membranes, as observed in the Fig. 7, due to the presence of an extra pore volume. As expected, the mesopores present in the MSS lead to a higher permeability, not only for ethanol but also for water. For the MSS-ZIF filled membranes, increase in the ethanol separation factors is very pronounced as compared to the unfilled PDMS 


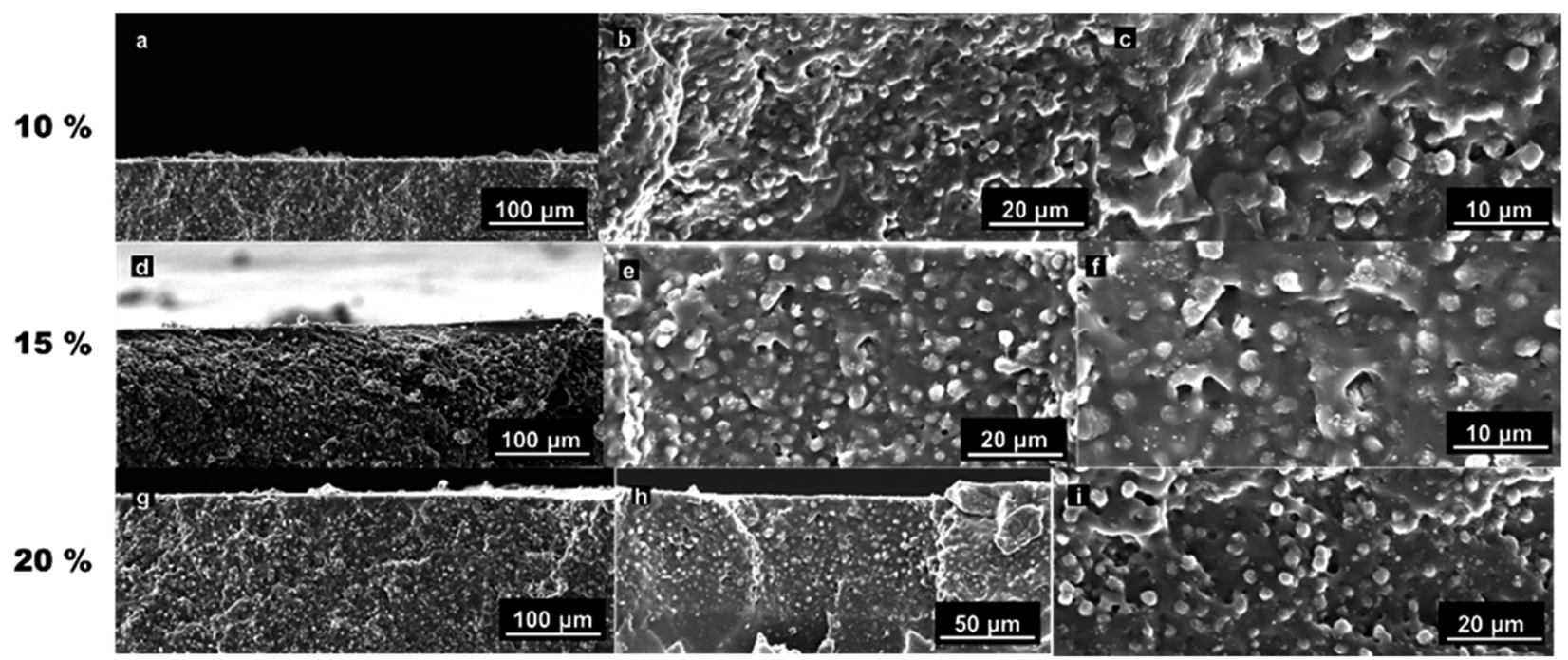

Fig. 6 Cross-sectional SEM micrographs of MMMs filled with MSS-ZIF-71 core-shell spheres: (a-c) 10 wt\%, (d-f) 15 wt $\%$, and (g-l) 20 wt $\%$ loading viewed at different magnifications.

membrane and much better than for the MSS filled membranes. This is attributed to the increased ethanol flux and reduced water flux for the MSS-ZIF filled membranes with increasing loadings due to its hydrophobic pore characteristic. As known from the adsorption isotherms, bigger pores of the MSS were sealed by the ZIF shell and the hydrophobic nature of ZIF-71 and ZIF-8 shells prevents the water molecules to adsorb and permeate. Similar observation has been reported by Ji and co-workers on the MIL-53 loaded PDMS membranes. ${ }^{80}$ Enhanced permeate flux, attributed to the hydrophobic surface and ethanol-affinity channels of MIL-53 particles, was observed for the PDMS membranes with MIL-53 loadings up to $40 \mathrm{wt} \%{ }^{80}$

Meanwhile, higher ethanol permeation can result from the higher affinity of ethanol for ZIF-71 and ZIF-8. It can be

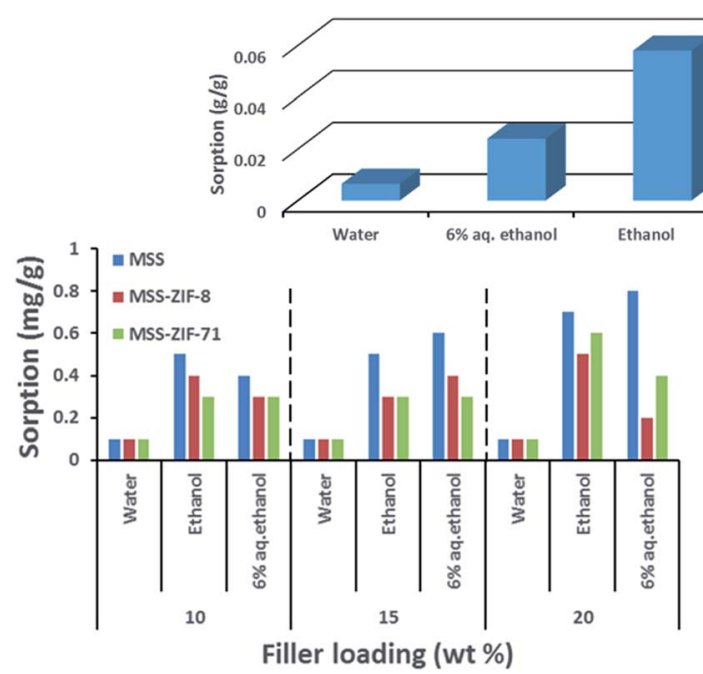

Fig. 7 Sorption in water, ethanol and the feed mixture for MSS, and MSS-ZIF filled PDMS MMMs with different filler loadings. The inset shows the sorption in water, ethanol and the feed mixture for unfilled PDMS membranes. observed from Fig. 8 that the normalized fluxes for the membranes filled with MSS-ZIF-71 are higher than the membranes filled with MSS-ZIF-8, while the separation factor is higher for the membranes filled with MSS-ZIF-8. This can be explained on the basis of the micropore window size of ZIF-71 $(0.48 \mathrm{~nm})^{81}$ which is larger than the one of ZIF-8 $(0.34 \mathrm{~nm}) .^{82}$ Thus might be responsible for some hydrogen bonded water to be adsorbed along with permeating ethanol molecules. Another possible reason for the higher normalized fluxes of the MSSZIF-71 filled membranes is the thinner ZIF-71 shell layer.

The incorporation of reference ZIF-8 and ZIF-71 materials into the PDMS network causes an important increase in the

(a)

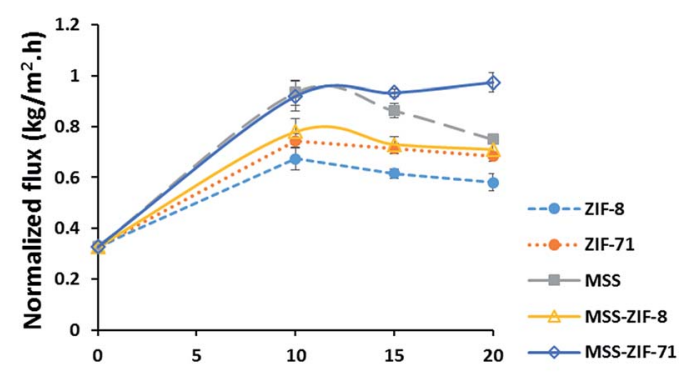

(b)

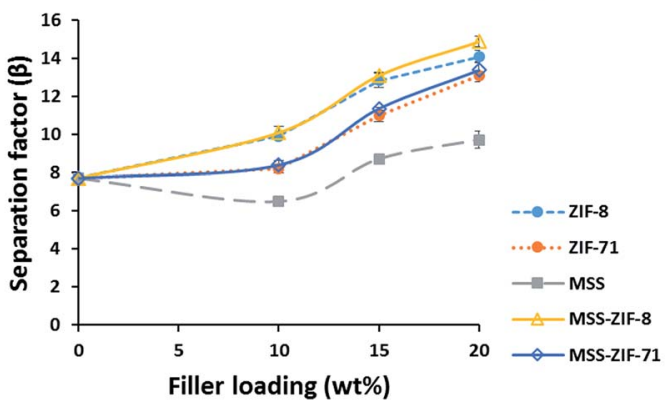

Fig. 8 Comparison of the pervaporation performance: (a) flux normalized to $10 \mu \mathrm{m}$ and (b) separation factor of the MSS-ZIF filled PDMS membranes at different filler loadings (10, 15 and 20 wt\%). 


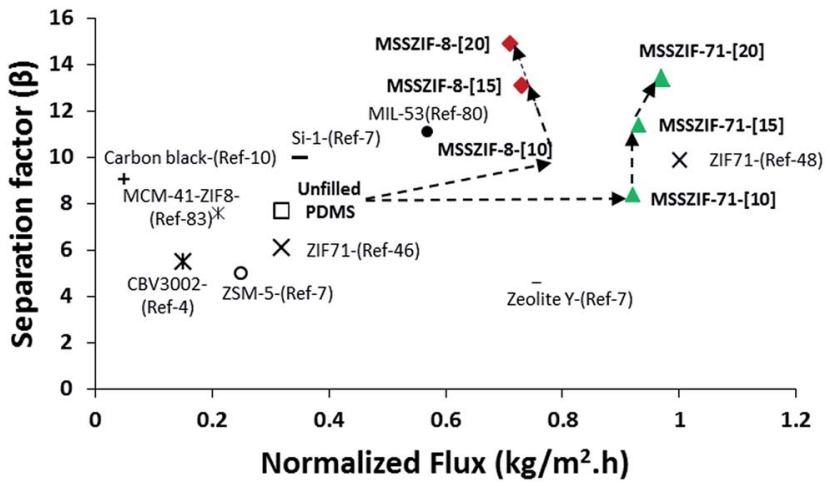

Fig. 9 Comparison with literature data for the pervaporation performance of MSS-ZIF- 8 and MSS-ZIF-71 MMMs with different loadings indicated between square brackets in $6 \mathrm{wt} \%$ water : ethanol separation at $40{ }^{\circ} \mathrm{C}$ (filler loadings of references: $10 \mathrm{wt} \%$ for carbon black and 30 wt\% for others).

ethanol selectivity. However, fluxes were low compared to MSS and MSS-ZIFs. Thus, the positive effect of the incorporation of the MSS-ZIFs as compared to mere ZIFs is especially striking for the flux. This confirms the expectations that the MSS core can provide a fast passage of the compound that passed the shell (i.e. ethanol). The selectivities of the MMMs with ZIFs and MSSZIFs almost coincide. This is indeed logical, since the extra selectivity upon filler incorporation is attributed to the good PDMS-filler interactions and the ethanol-selective sorption in the ZIF pores.

\section{Comparison with literature data}

Fig. 9 shows the pervaporation performance of the MSS-ZIF filled PDMS membranes prepared in this work with literature data using different kinds of fillers. It can be observed that the MSS-ZIF filled MMMs show a superior performance in the separation of ethanol from water with respect to flux and separation factor, outperforming PDMS membranes filled with other porous materials, such as zeolites, carbon black, MIL-53 (ref. 80) and MCM-41-ZIF-8 (ref. 83) composite. In our studies, the highest separation factor of 15 and a relatively high flux of $0.72 \mathrm{~kg} \mathrm{~m}{ }^{-2} \mathrm{~h}^{-1}$ was achieved for the PDMS membranes filled with $20 \mathrm{wt} \%$ of MSS-ZIF-8. Under similar conditions, $20 \mathrm{wt} \%$ MSS-ZIF-71 filled PDMS membranes gave a higher flux, approaching $1 \mathrm{~kg} \mathrm{~m}^{2} \mathrm{~h}^{-1}$, with a reasonable separation factor of 13. Thus, the improved separation performance of the MMMs demonstrated in this work could be related to the presence of micro- and mesoporous structure of the filler, where the mesoporous core facilitates the overall permeation of the specific component that is sorbed preferentially in the selective microporous ZIF shells. It is clear that with respect to MSS-ZIF fillers, ZIF-8 is the preferred coating when a high selectivity is necessary, while ZIF-71 is a better selection when higher fluxes are desired. A similar trend has also been reported on the pervaporation of $5 \%$ water : ethanol mixture using PDMS membranes loaded with an MCM-41-ZIF-8 composite. ${ }^{83}$ The prepared hybrid membranes showed an improved flux $(0.21 \mathrm{~kg}$ $\mathrm{m}^{-2} \mathrm{~h}^{-1}$ ) with a separation factor of 7.6 at $40{ }^{\circ} \mathrm{C} .{ }^{83}$

\section{Conclusions}

Uniform hierarchical micro- and mesostructures of MSS-ZIF-71 composites were prepared via control over crystallization kinetics in DMF solution. The introduction of dual micro- and mesostructures into the matrix of PDMS-based MMMs via MSSZIFs composite sphere incorporation significantly improved both fluxes and separation factors in the pervaporation of water : ethanol mixtures, compared to the unfilled and the MSS filled membranes. The MMMs filled with MSS-ZIF-71 gave excellent fluxes, while the MSS-ZIF-8 systematically gave high separation factors for similar loadings. The results clearly prove the potential of MSS-ZIFs filled MMMs for liquid phase separation processes, such as purification of bioethanol from fermentation broths.

\section{Acknowledgements}

The authors gratefully acknowledge Belgian Government (IAPPAI networking), the Flemish Government (Methusalem (CASAS) and FWO (G069811) and KU Leuven (IOFKP/10/002 and OT 11/061 projects)) for the financial support. L. H. W. $(12 \mathrm{M} 1415 \mathrm{~N})$ and S. T. (G004613N) thank the FWO-Vlaanderen for the postdoctoral research fellowship.

\section{References}

1 J. Gu, X. Shi, Y. Bai, H. Zhang, L. Zhang and H. Huang, Chem. Eng. Technol., 2009, 32, 155.

2 X. L. Liu, Y. S. Li, Y. Liu, G. Q. Zhu, J. Liu and W. S. Yang, J. Membr. Sci., 2011, 369, 228.

3 L. M. Vane, V. V. Namboodiri and T. C. Bowen, J. Membr. Sci., 2008, 308, 230.

4 A. Dobrak, A. Figoli, S. Chovau, F. Galiano, S. Simone, I. F. J. Vankelecom, E. Drioli and B. Van der Bruggen, J. Colloid Interface Sci., 2010, 346, 254.

5 G. P. Liu, F. J. Xiangli, W. Wei, S. N. Liu and W. Q. Jin, Chem. Eng. J., 2011, 174, 495.

6 I. F. J. Vankelecom, E. Scheppers, R. Heus and J. B. Uytterhoeven, J. Phys. Chem., 1994, 98, 12390.

7 I. F. J. Vankelecom, D. Depre, S. D. Beukelaer and J. B. Uytterhoeven, J. Phys. Chem., 1995, 99, 13193.

8 C. Dotremont, I. F. J. Vankelecom, M. Morobe, J. B. Uytterhoeven and C. Vandecasteele, J. Phys. Chem. B, 1997, 101, 2160.

9 X. Tang, R. Wang, Z. Xiao, E. Shi and J. Yang, J. Appl. Polym. Sci., 2007, 105, 3132.

10 I. F. J. Vankelecom, J. D. Kinderen, B. M. Dewitte and J. B. Uytterhoeven, J. Phys. Chem. B, 1997, 101, 5182.

11 S. Shi, Z. Du, H. Ye, C. Zhang and H. Li, Polymer, 2006, 38, 949.

12 B. Moermans, W. De Beuckelaer, I. F. J. Vankelecom, R. Ravishankar, J. A. Martens and P. A. Jacobs, Chem. Commun., 2000, 2467.

13 Ş. B. Tantekin-Ersolmaz, Ç. Atalay-Oral, M. Tatlıer, A. ErdemSenatalar, B. Schoeman and J. Sterte, J. Membr. Sci., 2000, $175,285$. 
14 J. P. Boom, I. G. M. Punt, H. Zwijnenberg, R. deBoer, D. Bargeman, C. A. Smolders and H. Strathmann, J. Membr. Sci., 1998, 138, 237.

15 K. S. Park, Z. Ni, A. P. Cote, J. Y. Choi, R. D. Huang, F. J. Uribe-Romo, H. K. Chae, M. O'Keeffe and O. M. Yaghi, Proc. Natl. Acad. Sci. U. S. A., 2006, 103, 10186.

16 Y. Liu, G. Zeng, Y. Pan and Z. Lai, J. Membr. Sci., 2011, 379, 46.

17 H. Bux, F. Y. Liang, Y. S. Li, J. Cravillon, M. Wiebcke and J. Caro, J. Am. Chem. Soc., 2009, 131, 16000.

18 Y. S. Li, F. Y. Liang, H. Bux, A. Feldhoff, W. S. Yang and J. Caro, Angew. Chem., Int. Ed., 2010, 49, 548.

19 S. R. Venna and M. A. Carreon, J. Am. Chem. Soc., 2010, 132, 76.

20 Y. S. Li, F. Y. Liang, H. Bux, W. S. Yang and J. Caro, J. Membr. Sci., 2010, 354, 48.

21 A. S. Huang, H. Bux, F. Steinbach and J. Caro, Angew. Chem., Int. Ed., 2010, 49, 4958.

22 T. Rodenas, I. Luz, G. Prieto, B. Seoane, H. Mira, A. Corma, F. Kapteijn, F. X. Llabrés i Xamena and J. Gascon, Nat. Mater., 2015, 14, 48.

23 B. Seoane, J. Coronas, I. Gascon, M. Etxeberria Benavides, O. Karvan, J. Caro, F. Kapteijn and J. Gascon, Chem. Soc. Rev., 2015, 44, 2421.

24 Y. Peng, Y. Li, Y. Ban, H. Jin, W. Jiao, X. Liu and W. Yang, Science, 2014, 346, 1356.

25 J. Yao and H. Wang, Chem. Soc. Rev., 2014, 43, 4470.

26 A. Phan, C. J. Doonan, F. J. Uribe-Romo, C. B. Knobler, M. O'Keeffe and O. M. Yaghi, Acc. Chem. Res., 2010, 43, 58.

27 K. S. Park, Z. Ni, A. P. Cote, J. Y. Choi, R. D. Huang, F. J. Uribe-Romo, H. K. Chae, M. O'Keeffe and O. M. Yaghi, Proc. Natl. Acad. Sci. U. S. A., 2006, 103, 10186.

28 S. Basu, M. Maes, A. Cano-Odena, L. Alaerts, D. E. De Vos and I. F. J. Vankelecom, J. Membr. Sci., 2009, 344, 190.

29 L. Ge, W. Zhou, A. Du and Z. Zhu, J. Phys. Chem. C, 2012, 116, 13264.

30 H. Bux, A. Feldhoff, J. Cravillon, M. Wiebcke, Y.-S. Li and J. Caro, Chem. Mater., 2011, 23, 2262.

31 M. C. McCarthy, V. Varela-Guerrero, G. V. Barnett and H.-K. Jeong, Langmuir, 2010, 26, 14636.

32 H. Bux, F. Liang, Y. Li, J. Cravillon, M. Wiebcke and J. Caro, J. Am. Chem. Soc., 2009, 131, 16000.

33 J. Yao, D. Dong, D. Li, L. He, G. Xu and H. Wang, Chem. Commun., 2011, 47, 2559.

34 Y. Pan and Z. Lai, Chem. Commun., 2011, 47, 10275.

35 H. T. Kwon and H.-K. Jeong, Chem. Commun., 2013, 49, 3854.

36 Z. Xie, J. Yang, J. Wang, J. Bai, H. Yin, B. Yuan, J. Lu, Y. Zhang, L. Zhou and C. Duan, Chem. Commun., 2012, 48, 5977.

37 L. Diestel, H. Bux, D. Wachsmuth and J. Caro, Microporous Mesoporous Mater., 2012, 164, 288.

38 H. Fan, Q. Shi, H. Yan, S. Ji, J. Dong and G. Zhang, Angew. Chem., Int. Ed., 2014, 53, 1.

39 G. M. Shi, T. X. Yang and T. S. Chung, J. Membr. Sci., 2012, 415-416, 577.

40 X. L. Liu, Y.-S. Li, G.-Q. Zhu, Y.-J. Ban, L.-Y. Xu and W.-S. Yang, Angew. Chem., Int. Ed., 2011, 50, 10636.
41 G. M. Sha, H. Chen, Y. C. Jean and T. S. Chung, Polymer, 2013, 54, 774.

42 R. Banerjee, A. Phan, B. Wang, C. Knobler, H. Furukawa, M. O'Keeffe and O. M. Yaghi, Science, 2008, 319, 939.

43 W. Morris, B. Leung, H. Furukawa, O. K. Yaghi, N. He, H. Hayashi, Y. Houndonougbo, M. Asta, B. B. Laird and O. M. Yaghi, J. Am. Chem. Soc., 2010, 132, 11006.

44 A. Nalaparaju, X. S. Zhao and J. W. Jiang, J. Phys. Chem. C, 2010, 114, 11542.

45 R. P. Lively, M. E. Dose, J. A. Thompson, B. A. McCool, R. R. Chance and W. J. Koros, Chem. Commun., 2011, 47, 8667.

46 X. L. Dong and Y. S. Lin, Chem. Commun., 2013, 49, 1196.

47 S. Liu, G. Liu, X. Zhao and W. Jin, J. Membr. Sci., 2013, 446, 181.

48 Y. Li, L. H. Wee, J. A. Martens and I. F. J. Vankelecom, J. Mater. Chem. A, 2014, 2, 10034.

49 L. H. Wee, Y. Li, K. Zhang, P. Davit, S. Bordiga, J. Jiang, I. F. J. Vankelecom and J. A. Martens, Adv. Funct. Mater., 2015, 25, 516.

50 Q. Zhang, I. Lee, J. B. Joo, F. Zaera and Y. Yin, Acc. Chem. Res., 2013, 46, 1816.

51 A. Burns, H. Ow and U. Wiesner, Chem. Soc. Rev., 2006, 35, 1028.

52 R. Haag, Angew. Chem., Int. Ed., 2004, 43, 278.

53 Z. Liu, M. Li, X. Yang, M. Yin, J. Ren and X. Qu, Biomaterials, 2011, 32, 4683.

54 Y. Diamant, S. Chappel, S. G. Chen, O. Melamed and A. Zaban, Coord. Chem. Rev., 2004, 248, 1271.

55 P. Strasser, S. Koh, T. Anniyev, J. Greeley, K. More, C. Yu, Z. Liu, S. Kaya, D. Nordlund, H. Ogasawara, M. F. Toney and A. Nilsson, Nat. Chem., 2010, 2, 454.

56 E. Biemmi, C. Scherb and T. Bein, J. Am. Chem. Soc., 2007, 129, 8054.

57 A. Huang, H. Bux, F. Steinbach and J. Caro, Angew. Chem., Int. Ed., 2010, 49, 4958.

58 K. Tanaka, T. Muraoka, D. Hirayama and A. Ohnish, Chem. Commun., 2012, 48, 8577.

59 S. Sorribas, B. Zornoza, C. Tellez and J. Coronas, Chem. Commun., 2012, 48, 9388.

60 C. Jo, H. J. Lee and M. Oh, Adv. Mater., 2011, 23, 1716.

61 A. Kudasheva, S. Sorribas, B. Zornoza and C. Téllez, J. Chem. Technol. Biotechnol., 2015, 90, 669.

62 T. S. Chung, L. Y. Jiang, Y. Li and S. Kulprathipanja, Prog. Polym. Sci., 2007, 32, 483.

63 C. M. Zimmerman, A. Singh and W. J. Koros, J. Membr. Sci., 1997, 137, 145.

64 B. Adnadjevic, J. Jovanovic and S. Gajinov, J. Membr. Sci., 1997, 136, 173.

65 I. F. J. Vankelecom, C. Dotremont, M. Morobe, J. B. Uytterhoeven and C. Vandecasteele, J. Phys. Chem. B, 1997, 101, 2154.

66 S. R. Venna, J. B. Jasinski and M. A. Carreon, J. Am. Chem. Soc., 2010, 132, 18030.

67 J. Cravillon, C. A. Schröder, H. Bux, A. Rothkirch, J. Caro and M. Wiebcke, CrystEngComm, 2012, 14, 492. 
68 S. Springer, A. Satalov, J. Lippke and M. Wiebcke, Microporous Mesoporous Mater., 2015, 216, 161.

69 Y. Bouizi, I. Daiz, L. Rouleau and V. P. Valtchev, Adv. Funct. Mater., 2005, 15, 1955.

70 A. Schejn, L. Balan, V. Falk, L. Aranda, G. Medjahdi and R. Schneider, CrystEngComm, 2014, 16, 4493.

71 H. Cölfen and S. Mann, Angew. Chem., Int. Ed., 2003, 42, 2350.

72 Y. Yin and A. P. Alivisatos, Nature, 2005, 437, 664.

73 T. A. Peters, J. Fontalvo, M. A. G. Vorstman, N. E. Benes, R. A. van Dam, Z. A. E. P. Vroon, E. L. J. van SoestVercammen and J. T. F. Keurentjes, J. Membr. Sci., 2005, 248, 73.

74 R. Ameloot, E. Gobechiya, H. Uji-i, J. A. Martens, J. Hofkens, L. Alaerts, B. F. Sels and D. E. De Vos, Adv. Mater., 2010, 22, 2685.

75 N. Masoumifard, P. M. Arnal, S. Kaliaguine and F. Kleitz, ChemSusChem, 2015, 8, 2093.
76 L. Jia, X. Sun, X. Ye, C. Zou, H. Gu, Y. Huang, G. Niu and D. Zhao, Microporous Mesoporous Mater., 2013, 176, 16.

77 X. F. Qian, B. Li, Y. Y. Hu, G. X. Niu, D. Yahong, H. Zhang, R. C. Che, Y. Tang, D. S. Su, A. M. Asiri and D. Y. Zhao, Chem.-Eur. J., 2012, 18, 931.

78 F. Zhang, Y. Yan, Y. Meng, Y. Xia, B. Tu and D. Zhao, Microporous Mesoporous Mater., 2007, 98, 6.

79 J. Lee, J. Kim and T. Hyeon, Chem. Commun., 2003, 1138.

80 G. Zhang, J. Li, N. Wang, H. Fan, R. Zhang, G. Zhang and S. Ji, J. Membr. Sci., 2015, 492, 322.

81 K. Zhang, R. P. Lively, M. E. Dose, A. J. Brown, C. Zhang, J. Chung, S. Nair, W. J. Koros and R. R. Chance, Chem. Commun., 2013, 49, 3245.

82 X. C. Huang, Y. Y. Lin, J. P. Zhang and X. M. Chen, Angew. Chem., Int. Ed., 2006, 45, 1557.

83 N. Wang, G. Shi, J. Gao, J. Li, L. Wang, H. Guo, G. Zhang and S. Ji, Sep. Purif. Technol., 2015, 153, 146. 